\title{
Sistemas de Visualización Gamificados para la mejora de la Motivación Intrínseca en Estudiantes de Arquitectura Gamified Visual Systems for improving the Intrinsic Motivation of Architectural Students
}

\author{
David Fonseca $^{1}$, Isidro Navarro ${ }^{1}$, Sergi Villagrasa ${ }^{1}$, Francesc Valls ${ }^{2}$, Ernest Redondo ${ }^{2}$, Marc Gené ${ }^{1}$, Xavi Calvo ${ }^{3}$, \\ Claudia Aymerich ${ }^{3}$, Miquel Sans ${ }^{4}$ \\ \{fonsi; inavarro; sergiv; mgene\}@salle.url.edu ${ }^{1}$, \{ernesto.redondo; francesc.valls\}@upc.edu², \\ \{xavicalvof; clau.ayme\}@gmail.com, miquelet-sans@hotmail.com \\ ${ }^{1,3,4}$ La Salle Campus Barcelona \\ Universitat Ramon Llull \\ Barcelona, España \\ ${ }^{2}$ AR\&M, Arquitectura Barcelona, BarcelonaTech \\ Universidad Politécnica de Catalunya \\ Barcelona, España
}

\begin{abstract}
Resumen- El artículo presenta y analiza los resultados obtenidos en el proceso de incorporar sistemas gamificados de visualización 3D para proyectos urbanos en los estudios de grado y máster de Arquitectura. El proceso se ha diseñado a partir de una primera de recogida de datos iniciales para evaluar el perfil de los estudiantes, un segundo proceso práctico de formación y trabajo en entornos urbanos reales con el fin de diseñar nuevos usos a partir de una reordenación del espacio, todo ello utilizando sistemas digitales interactivos con el fin de gamificar la experiencia, y una tercera fase de recogida de resultados ligados a motivación, satisfacción y usabilidad de la experiencia. Los datos presentados se centran en la motivación inicial recogida de los estudiantes. Los resultados nos revelan una baja motivación inicial del estudiante, no entendiendo las utilidades que los juegos y entornos virtuales pueden aportar a la visualización del proyecto arquitectónico. No obstante, la propuesta utilizada ha conseguido mejorar dicha percepción inicial, mejorando el interés por la materia y en definitiva en las competencias de trabajo en equipo y en presentación tridimensional de proyectos.
\end{abstract}

Palabras clave: educación arquitectónica, motivación, gamificación, realidad virtual, aprendizaje basado en proyectos, aprendizaje colaborativo.

\begin{abstract}
The article analyzes the results obtained in the process of incorporating gamified 3D visual systems for urban projects into the degree and master of architecture. The process has been designed through a first collection of profile student's data, followed by the practical process of working in real urban environments, and finally collecting the results lined to motivation, satisfaction and usability of the process. This project seeks to design new applications from a rearrangement of urban space using gamify-interactive digital systems in order to improve the user experience. The results reveal a low initial motivation in the students, not understanding that the usefulness of the games and the virtual environments can contribute to the visualization of the architectural and urban project. Nevertheless, the proposal has improved the initial perception, raising the interest in the subject and, ultimately, the teamwork skills and the three-dimensional presentation of the project.
\end{abstract}

Keywords: architectural education, motivation, gamification, virtual reality, project based learning, collaborative learning.

\section{INTRODUCCIÓN}

La formación en general y la arquitectónica no está exenta de un proceso de cambio derivado de los nuevos sistemas visuales digitales e interactivos (Kreijns, Van Acker, Vermeulen, \& Van Buuren, 2013). Estos sistemas han revolucionado los métodos clásicos basados en el dibujo a mano alzada, la representación en dos dimensiones de planos y fotomontajes, y las maquetas físicas (Boeykens, Santana Quintero, \& Neuckermans, 2008). Estos procesos tienen un flujo de trabajo muy lento y caro, ya que cualquier cambio supone no solo modificar planos, sino re-imprimirlos, por no hablar de lo que suponen los cambios a realizar en las maquetas o la dificultad de incluir representaciones complejas de materiales y texturas.

Gracias primero a los sistemas CAD (Computer Aided Design), y actualmente a los sistemas BIM (Building Information Modelling) que funcionan de forma paramétrica, sin olvidarnos de las nuevas tecnologías como la Realidad Aumentada (RA), la Realidad Virtual (RV), o la Gamificación, en la actualidad el proyecto arquitectónico se puede visualizar, cambiar y actualizar en diversos formatos mediante un flujo de trabajo muy rápido, barato y que permiten una comprensión del espacio incluso mejor que con los citados sistemas tradicionales (Vicent, Villagrasa, Fonseca, \& Redondo, 2015). A medida que se trabaja con un proyecto urbano, de escala habitualmente mucho mayor que el arquitectónico, los detalles y/o calidades de los elementos hacen todavía más crítico controlar el flujo de trabajo, siendo necesario que el progreso del proyecto pueda estudiarse rápidamente para generar las modificaciones oportunas lo antes posible y no encarecer la realización del mismo.

El presente artículo se enmarca dentro de las líneas de trabajo de un proyecto del programa Retos del Ministerio de Educación y Competitividad de España (2016-2020), con el fin de mejorar la docencia en el campo de la Arquitectura mediante nuevos métodos que implican en uso de tecnologías visuales inmersivas y colaborativas, todo ello en un ambiente gamificado. Esta aproximación busca no solo mejorar la docencia mediante un aumento en la motivación del 
estudiante, sino incorporar la respuesta del usuario final al diseño del proyecto, un aspecto que se engloba dentro de la actual tendencia denominada Educación Informal y que mejora determinadas competencias sociales y en uso de las TIC (Tecnologías de la Información y la Comunicación) de los actuales estudiantes.

\section{CONTEXTO}

\section{A. El proyecto urbano y su educación gamificada}

El uso y dominio de las TIC (Tecnologías de la Información y la Comunicación), es un reto de la sociedad actual, tanto a nivel formativo, como a nivel profesional y finalmente en cualquier capa de la sociedad, tanto urbana como rural (Oliver, 2002). Dicho reto es complicado de conseguir en sí mismo debido a diversos factores como por ejemplo: la alta velocidad en la aparición, mejora, e incluso desaparición de todo tipo de tecnologías, los elevados costes de alguna de ellas, la dificultad de uso que conllevan, o simplemente la falta de oportunidades para que determinadas TIC se adapten de forma generalizada a todos los niveles de la sociedad, por costes o simplemente soporte de uso.

Centrados en el proyecto urbano, no solo a nivel profesional, sino también educativo, las estrategias de aproximación han sido históricamente clásicas y poco dinámicas. Lo más normal ha sido el uso de paneles informativos, maquetas, infografías, o videos. Estas estrategias no contemplan la interacción con el usuario, una posibilidad que las actuales TIC comienzan a permitir de forma amplia, incluso con un enfoque gamificado. Las estrategias basadas en juegos serios que plantean retos colaborativos para llegar a soluciones de consenso, no solo son interesantes desde un punto de vista de democracia ciudadana, sino que a nivel educativo aumentan la motivación del estudiante y por consiguiente su rendimiento académico (David Fonseca, Valls, Redondo, \& Villagrasa, 2016; Hjorth \& Wilensky, 2014; Redondo et al., 2014).

Las estrategias gamificadas, aparte de seguir las reglas de diseño lógicas de cualquier sistema gamificado, deben ser capaces de incluir herramientas y opciones específicas para la interacción de los proyectos arquitectónicos y urbanos, en base a los comentarios y experiencias de los estudiantes y los usuarios finales. Existen referentes del uso de la gamificación en procesos de planificación urbanística unidos a otros de participación ciudadana como es el caso del "Blockholm" (Estocolmo, 2014), un juego basado en Minecraft promovido por el Swedish Center for Architecture and Design de Estocolmo que contó con la participación de hasta 100.000 usuarios, técnicos y expertos en diseño urbano y ciudadanos. En este caso, el objetivo del juego se basa en diseñar una ciudad del futuro inteligente a partir de la cartografía real de la ciudad donde se incluyen la topografía, las calles, las parcelas, los ríos, los lagos etc (ver Fig. 1).

Otros ejemplos son el "Play the City", de la Play the City Foundation implementado a lo largo del 2012 en diferentes ciudades de Holanda, Bélgica, Turquía o Sudáfrica y el cual estaba basado sobre un juego tipo Word of Warcraft. Otras estrategias utilizadas previamente se han basado en el uso del juego "SimCity", en sus diversas versiones, en talleres de planificación urbana, destacando el caso de Ciudad el Cabo en el 2013 (ver Fig.1).

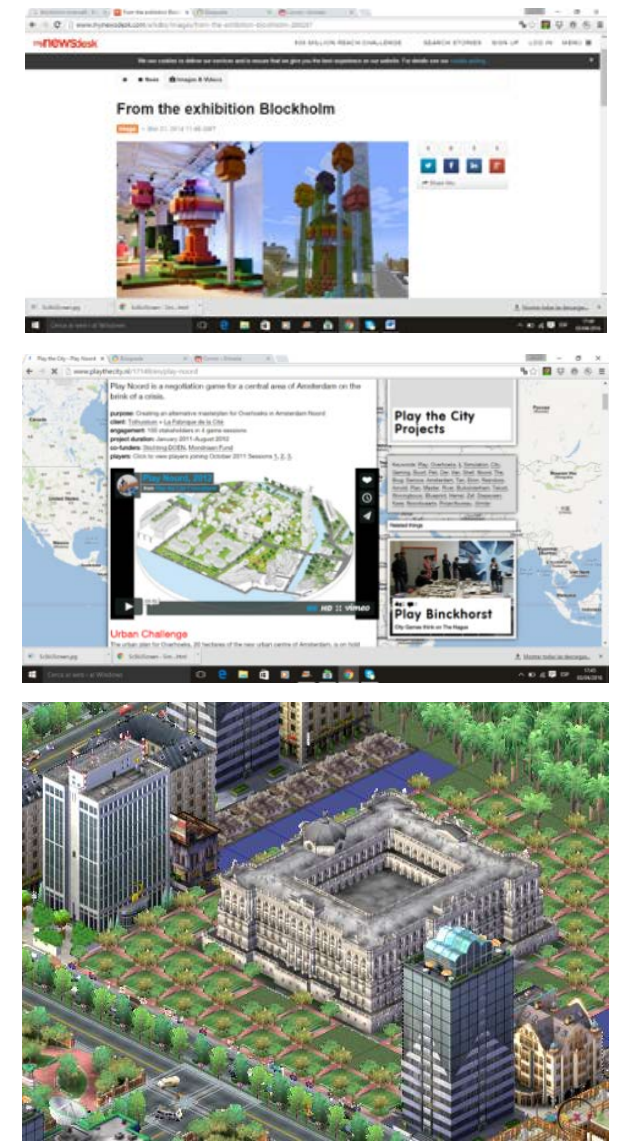

Figura 1. Ejemplos del Proyecto Blockholm, Play the City y SimCity 3000.

Aunque Blokcholm llegó a diseñar espacios reales que fueron reconstruidos a escala $1 / 5$, el tema del diseño urbano no se abordó en ninguno de ellos más allá de un simple cambio de escala en el modelo virtual generado. Son propuestas básicas de zonificación, de usos generales a nivel de toda una urbanización, o de trabajo digital a gran escala. En todos ellos se incide en el planeamiento urbano, mientras que en la propuesta actual, se busca trabajar sobre el proyecto arquitectónico y el diseño urbano a una escala que permita mayor nivel de detalle y complejidad.

\section{B. Aprendizaje 3.0: el uso de tecnologías en educación formal e informal.}

Tal y como demuestran multitud de recientes investigaciones, el uso de dispositivos móviles, wearables tecnologías como la RV, la RA, el trabajo deslocalizado y colaborativo, y estrategias gamificadas están calando en nuestra sociedad gracias a su facilidad de uso y eficacia, tanto en etapas formativas, como posteriormente en ámbitos profesionales y sociales (Valls, Redondo, \& Fonseca, 2015; Vicent et al., 2015). Mediante el uso de estas tecnologías podemos trabajar con propuestas urbanas definidas ensayando diversas estrategias de actuación de forma interactiva. De esta forma podemos evaluar de forma colaborativa los espacios públicos gracias a la colaboración de todos los agentes descritos previamente (estudiantes, profesionales y usuarios finales) (David Fonseca et al., 2016). Estas incorporaciones permiten definir los llamados proyectos 3.0: el usuario final, la ciudadanía, y el estudiante, dejan de ser actores pasivos para participar el proceso de diseño del proyecto. La nueva metodología establece una estrategia de aprendizaje basado en 
proyectos (o Project Based Learning, PBL), pero incluso actuando con un rol concreto que le permita obtener y mejorar sus competencias espaciales y sociales de forma mucho más óptima (estrategia conocida como SCC, Scenario Centered Curriculum), tanto de manera formal (con los contenidos a desarrollar en clase) como informal (con las opiniones de los usuarios finales que permiten mejorar no solo el proyecto sino también la formación.

Partiendo de un enfoque crítico, la primera pregunta que nos podríamos hacer es: ¿Qué aportan los juegos serios a estos procesos? Las respuestas son múltiples: En primer lugar, las estrategias de juego se han demostrado como un elemento de aprendizaje o de toma de decisiones muy interesantes, útiles y aplicables en diversas áreas de conocimiento (marketing, negocios, educación formal, educación no formal, etc.). Por otro lado, otras respuestas sobre la utilidad de los juegos serios las podemos obtener en las actuales demostraciones sobre el aporte de un grado de motivación superior en el seguimiento de los contenidos gamificadas, el incremento de la participación que suponen, y por supuesto en el dinamismo inherente que aportan a dichos contenidos (D Fonseca, Martí, Redondo, Navarro, \& Sánchez, 2014; Redondo et al., 2014).

La experimentación del uso de los juegos serios en los procesos de participación ciudadana debe permitir ahondar en la compresión de la ciudad-barrio-espacio público, un espacio físico concreto como lugar de comunicación en el que se generan iniciativas de todo tipo. La gamificación de un espacio real genera un espacio virtual y un entorno urbano de simulación en el que es posible hacer experimentos dinámicos de participación y generación de ideas, usos o cambios que mejoren dicho espacio.

\section{Evaluación de la motivación}

La necesidad de definir una evaluación en cualquier estudio radica en la validación en sí misma del trabajo. Mediante el diseño de un Pre-Test se consiguen obtener datos tan importantes como el perfil de usuario y/o los niveles iniciales de las variables de trabajo. Mediante el perfil del estudiante se puede comprobar rápidamente si están o no preparados para seguir el curso y/o utilizar las tecnologías diseñadas. Un grupo sin acceso a las tecnologías previstas, por muy motivado que esté, puede degenerar un fracaso de implantación educativa, siendo necesario rediseñar el método adaptado a las posibilidades de los estudiantes. Mediante la evaluación de los niveles iniciales de determinadas variables se puede comprobar en la fase final la evolución obtenida mediante el método propuesto (Bienkowski, Feng, \& Means, 2012).

En las etapas finales el trabajo con los Post-Test podemos evaluar las propuestas desde diversos ámbitos, como por ejemplo el grado de usabilidad, satisfacción, competencia o motivación que la experiencia ha generado en el usuario (en nuestro caso el alumno). Los enfoques pueden ir desde trabajos cuantitativos, cualitativos y mixtos (donde se mezclan ambos sistemas para categorizar con más detalle las respuestas a las variables de estudio).

El estudio de la motivación es relevante y significativo, tanto en cuanto se ha demostrado previamente (David Fonseca, Redondo, \& Villagrasa, 2014), como un estudiante motivado puede mejorar mucho más rápido y eficientemente independientemente de su nivel inicial. Este aspecto ligado con el ámbito de estudio (el grado de Arquitectura), confiere al presente trabajo un interés singular, dado que los estudios de Arquitectura en España están ligados a la vertiente más técnica de la Educación Superior, y más alejada de la rama artística, como sucede en otros países. Por dicho motivo, los últimos años y debido a la crisis del sector (Peña, Fonseca, \& Martí, 2016), el aumento de la internacionalización y la inherente dificultad no esperada ni conocida muchas veces de dichos estudios, hace provocado un descenso en el número de alumnos así como tasas de abandono elevadas. En definitiva, queda claro que evaluar el grado de motivación y utilizar métodos diferenciados que permitan mantener una tasa elevada de empatía entre el alumno y la materia es fundamental para no perder vocaciones en fases tempranas de los estudios.

En este campo, uno de los métodos más utilizados y validados para la medición de la motivación es el IMI (Intrinsic Motivation Inventory, 1994). El IMI permite evaluar seis niveles principales relacionados con el grado de interés/disfrute, nivel de competencia, esfuerzo, utilidad, presión/tensión y grado de cambio percibido dada una actividad. A estas seis escalas, se ha añadido recientemente un séptimo nivel (relación), aunque su validez debe todavía ser definida de forma estricta.

De los siete niveles, la escala correspondiente a interés/disfrute se considera en sí misma como la valorativa de la motivación intrínseca, de forma que aunque el sistema recibe dicho nombre, solo una de las variables evalúa el aspecto citado. Los otros niveles se utilizan habitualmente como predictores. Por ejemplo, el concepto de competencia percibida se asocia a un predictor positivo, mientras que el de presión/tensión se asocia a un predictor negativo de la motivación intrínseca. Otros niveles como el de esfuerzo se consideran un elemento independiente, mientras que la utilidad se suele utilizar en estudios internacionales para relacionarla y autorregularla con las actividades diferenciadas de cada ámbito de estudio. Finalmente la sub-escala de relación se utiliza en estudios que tienen que ver con las interacciones interpersonales y el trabajo colaborativo.

\section{DESCRIPCIÓN}

El estudio descrito en el presente artículo se centra en la descripción del proceso de implementación de sistemas gamificados de RV en la enseñanza de arquitectura y la percepción de utilidad y motivación del estudiante delante de dichas propuestas. El objetivo global del proyecto trata de recrear virtualmente zonas urbanas de la ciudad de Barcelona y alrededores para establecer un entorno gamificado donde los usuarios (estudiantes, profesionales del sector, arquitectos y ciudadanos de todo tipo y edades), puedan interactuar (jugar), con el fin de recrear nuevos espacios centrados en usos peatonales, en lugar de los actuales que no contemplan predominantemente esta tipología de espacio. El espacio tridimensional virtual se pretende adicionalmente que tenga una calidad fotográfica (es decir de máximo realismo), incorporando al ambiente los movimientos, materiales, texturas e incluso sonidos propios de dicho entorno.

En la fase actual de creación de un proyecto piloto las tareas en realización y su distribución son:

- Definición del entorno urbano, propuesta inicial de usos, modelado y texturización de edificios (UPC). Todo ello 
mediante herramientas de modelado tipo 3DMax, Rhyno, Sketchup, Photoshop, etc (ver ejemplo de Fig.2).

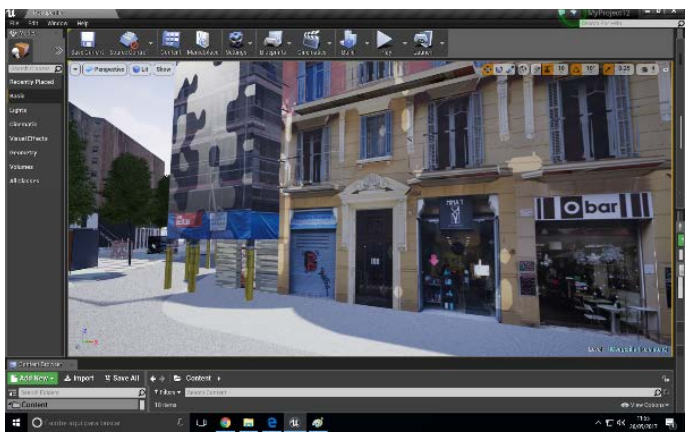

Figura 2. Integración modelos 3D de edificios en entorno de Unreal.

- Definición de las mecánicas de juego, interacción, restricciones y sistema de implementación, programación e integración de modelos e interfaz (La Salle). Para este proceso se está utilizando Unreal (ver ejemplo en Fig. 3).

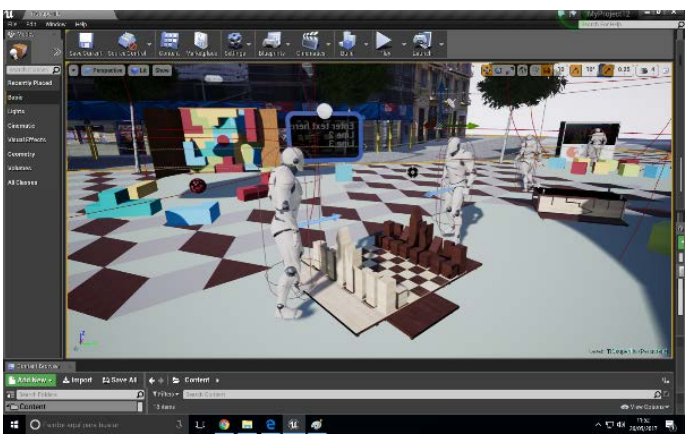

Figura 3. Inclusión de personajes y programación de acciones y retos en Unreal.

- Definición de métricas de evaluación y análisis (La Salle). Ámbitos de estudio: Asignatura de Representación Multimedia, grado Arquitectura UPC; Máster de Paisajismo UPC; Asignatura de Herramientas de Representación II, grado Arquitectura y Arquitectura Técnica La Salle.

Los procesos descritos se han realizado en el segundo semestre del curso académico 2016-2017, siendo los modelos provisionales, y a la espera de obtener los resultados del posttest una vez cerrado el curso.

Para el estudio, hemos utilizado un cuestionario inicial para obtener por un lado el perfil tecnológico de los estudiantes y el grado de interés en las temáticas y enfoque del curso. Para el apartado del perfil, se han preguntado datos estadísticos como la edad, género, lugar de nacimiento, estudios acabados y en curso y posible experiencia laboral. Así mismo se han preguntado sobre los dispositivos y acceso a servicios de Internet tanto a nivel de utilización/pertenencia como frecuencia de uso.

En un segundo bloque hemos incluido un total de 18 preguntas divididas en tres bloques (ver Tabla 1). El primero de ellos (formado por ocho preguntas identificadas como A1A8), se ha centrado en obtener la percepción por parte del alumno del grado de utilidad de tecnologías como la RA/RV para la visualización y comprensión del espacio arquitectónico. El segundo bloque de tres preguntas (G1-G3), se han centrado en evaluar la percepción de utilidad que tienen los juegos en la comprensión del espacio y su uso educativo. Finalmente, mediante 7 preguntas (identificadas como IM1IM7), hemos evaluado la respuesta subjetiva de los estudiante referidas al IMI.

Tabla 1. Variables de control Pre-Test.

\begin{tabular}{|c|c|}
\hline Pregunta & Variable \\
\hline $\begin{array}{l}\text { La visualización digital en 3D de proyectos arquitectónicos y } \\
\text { urbanos es de vital importancia para su comprensión }\end{array}$ & A1 \\
\hline $\begin{array}{l}\text { El uso de realidad virtual para visualizar proyectos es útil para } \\
\text { su comprensión }\end{array}$ & A2 \\
\hline $\begin{array}{l}\text { El uso de realidad aumentada para visualizar proyectos es útil } \\
\text { para su comprensión }\end{array}$ & A3 \\
\hline $\begin{array}{l}\text { Estoy motivado para utilizar RA/RA en la fase de presentación } \\
\text { de proyectos }\end{array}$ & A4 \\
\hline $\begin{array}{l}\text { Los materiales, texturas e iluminación de una escena virtual } \\
\text { deben ser siempre lo más realísticos posibles }\end{array}$ & A5 \\
\hline Los sonidos ambientales deben ser lo más realísticos posibles & A6 \\
\hline $\begin{array}{l}\text { La existencia de música ambiental me satisface en la } \\
\text { visualización e interacción con propuestas virtuales }\end{array}$ & A7 \\
\hline $\begin{array}{l}\text { El dispositivo de visualización (móvil, tableta, ordenador, } \\
\text { gafas) influye mucho en la percepción de la calidad virtual }\end{array}$ & A8 \\
\hline $\begin{array}{l}\text { La utilización de un entorno gamificado (con misiones y } \\
\text { logros) es mejor que la navegación libre por zonas virtuales }\end{array}$ & G1 \\
\hline $\begin{array}{l}\text { En el caso de sistemas de juegos, me motivan más aquellos que } \\
\text { son unipersonales respecto a los multi-jugador }\end{array}$ & G2 \\
\hline $\begin{array}{l}\text { La utilización de juegos en ámbitos educativos considero que } \\
\text { aportan una mejor compresión de las materias }\end{array}$ & G3 \\
\hline $\begin{array}{l}\text { Disfruto jugando en entornos virtuales. Considero este tipo de } \\
\text { experiencias muy divertidas y entretenidas }\end{array}$ & IM1 \\
\hline $\begin{array}{l}\text { Mediante la visualización 3D adquiero mejor competencias } \\
\text { ligadas a la arquitectura respecto sistemas tradicionales }\end{array}$ & IM2 \\
\hline $\begin{array}{l}\text { Considero que el uso de propuestas virtuales y gamificadas } \\
\text { necesitan de menor esfuerzo que los sistemas tradicionales }\end{array}$ & IM3 \\
\hline $\begin{array}{l}\text { El uso de propuestas virtuales me genera menos tensión, stress, } \\
\text { nerviosismo que sistemas basados en maquetas y paneles }\end{array}$ & IM4 \\
\hline $\begin{array}{l}\text { Consideras que utilizando propuestas virtuales cambias tu } \\
\text { forma de trabajar en el futuro con presentaciones } \\
\text { arquitectónicas? }\end{array}$ & IM5 \\
\hline $\begin{array}{l}\text { Este tipo de actividades (juegos y navegación virtual), son } \\
\text { útiles para mi futuro y me pueden beneficiar }\end{array}$ & IM5 \\
\hline $\begin{array}{l}\text { Estos sistemas me ayudan a interrelacionarme con otros } \\
\text { usuarios/compañeros/amigos ampliando mis relaciones }\end{array}$ & IM7 \\
\hline
\end{tabular}

\section{Resultados}

La muestra de trabajo han sido un total de 75 alumnos, 30 varones y 35 mujeres, con una edad media de 23.8 años (Desviación Típica, DT: 5.47). El 56\% son españoles y el resto de múltiples nacionalidades: 5 noruegos, 3 colombianos, 2 franceses, venezolanos, ecuatorianos, y un representante de México, Italia, Austria, Argentina, Costa Rica, Bulgaria, Perú, Brasil, Estados Unidos de América y Andorra. 9 de ellos estaban cursando nivel de Master y el resto, 66, el nivel de grado. Solo el 16\% estaba alternando sus estudios con algún tipo de trabajo.

Revisando el perfil tecnológico de dispositivos, usos y frecuencias de utilización (según escala de Likert de 5 niveles donde el 1 equivale a "Nunca”, y el 5 "Muy frecuentemente”), el dispositivo más utilizado es el Smartphone (Promedio de 4.8), seguido del ordenador portátil (4.5). A mayor distancia encontramos el ordenador de sobremesa y las cámaras digitales de foto (2.9), el GPS (Global Position System, con un 2.7), o la Tableta (2.5). Otros dispositivos como cámaras digitales de video, teléfonos convencionales, relojes inteligentes o bandas de control no superan el 2/5. 
En cuanto a los usos de Internet, las Redes Sociales se sitúan en primer lugar destacadamente (4.3), seguidas por las búsquedas relacionadas con Arquitectura (3.9), y el visionado de noticias o información general (3.7). Con un 4.3 los estudiantes se conectan mediante cable/fibra óptica en casa, seguida por la conexión WIFI en lugares públicos (3.9), WIFI en casa (3.4), y conexiones en ordenadores de la universidad y/o el trabajo (3.3). El consumo de Internet en puestos fijos se sitúa de promedio entre 2-3 horas al día, seguido por los juegos o videojuegos en dispositivos móviles (con un promedio de 2 horas diarias), valor superior al tiempo de navegación por Internet en móvil (menos de 1h diaria), o del tiempo dedicado a videojuegos con consolas (entre 1 y $2 \mathrm{~h}$ de promedio diario).

Estos datos del perfil tecnológico de los alumnos nos reflejan un elevado consumo de productos por Internet ligados a su ámbito de estudio/trabajo (la arquitectura), y una predisposición al juego elevada (tanto en plataformas fijas como en móviles, especialmente en este último caso).

En la Figura 4, podemos ver gráficamente el resultado de las variables de estudio presentadas en la Tabla 1.

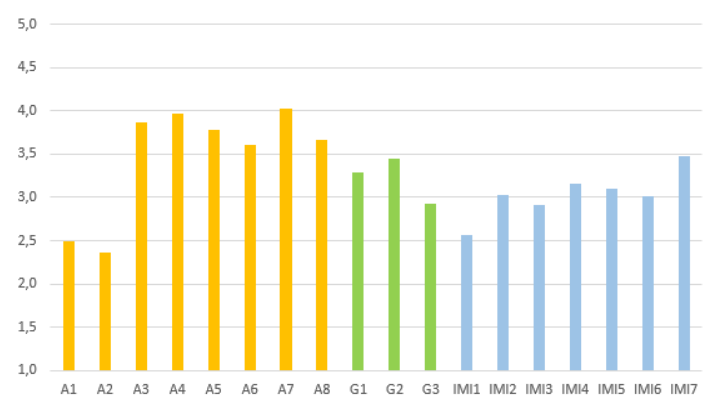

Figura 4. Variables de estudio (Pre-test, global).

Del primer bloque de cuestiones generales analizadas [A], el promedio obtenido ha sido de 3.5, habiendo ponderado a la baja las dos primeras cuestiones (A1: 2.5 y A2: 2.4), que corresponden a las afirmaciones de lo importante que es la visualización 3D de proyectos arquitectónicos para su comprensión y en la misma línea, que el uso de la RV es útil para dicha comprensión. Centrados en el bloque de gamificación [G], el promedio de las tres variables estudiadas ha sido de 3.2. Este valor refleja un interés medio, destacando en la zona inferior con un 2.9 la posible utilidad que los estudiantes ven a los juegos en ámbitos educativos.

El último bloque centrado en la medición del IMI ha obtenido un promedio de 3.0. El aspecto mejor valorado con un 3.5 ha sido el IMI-7 (los sistemas TIC ayuda a la relación personal con mis compañeros), y el menor el IMI-1 (disfrute del juego como experiencia divertida y entretenida).

Una vez realizado el curso, en el que los alumnos han tenido que realizar las propuestas urbanas para la peatonalización de unas calles concretas de Barcelona (ver Figura 5) y las han tenido que integrar en el sistema interactivo para su visualización (Figura 6), se ha realizado un post test en el que además de repetir las variables iniciales, se les ha preguntado sobre determinados aspectos ligados a la usabilidad y satisfacción con el método propuesto.

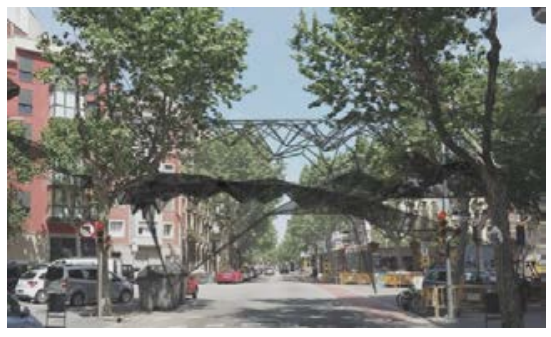

Figura 5. Propuesta cubierta móvil.

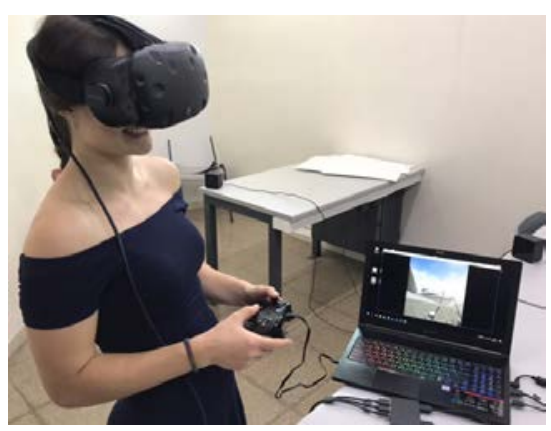

Figura 6. Ejemplo de navegación/interacción.

En la Figura 7, podemos ver gráficamente la comparativa de la evolución obtenida por las variables analizadas tanto en el Pre-test como en Post-test.

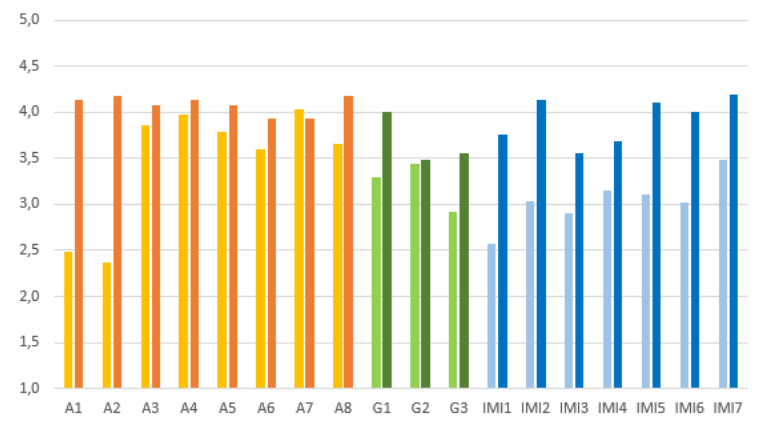

Figura 7. Variables de estudio (Comparativa Pre vs Post).

Como se puede observar, prácticamente todas las variables estudiadas de los tres índices (Globales [A], Gamificación [G] y Motivación [IMI]), han obtenido una mejor valoración en la finalización del curso, con incrementos significativamente estadísticos en los tres índices (pasando A del 3.5 al 4.1, G del 3.2 al 3.7, y finalmente el IMI de 3.0 al 3.9, siendo el incremento más elevado).

Así mismo se ha obtenido un grado de satisfacción de 3.98/5 en base a tres variables de trabajo: Me ha gustado utilizar sistemas de render en tiempo real, me ha gustado utilizar sistemas de visualización AR/VR y me ha gustado utilizar sistemas gamificados interactivos de visualización arquitectónica

Por el contrario, la usabilidad del sistema se ha situado en un 3.39/5, valor que podríamos considerar como aceptable pero no especialmente óptimo. Las tres preguntas de trabajo se han basado en las de satisfacción, substituyendo "Me ha gustado”, por “Me ha sido fácil”.

\section{CONCLUSIONES}

El presente estudio analiza el impacto de incluir nuevos sistemas de visualización interactivos y gamificados en el 
desarrollo del proyecto urbano, todo ello a nivel educativo tanto en grado como máster de arquitectura. Si bien el proyecto financiado en el cual se enmarca el trabajo se encuentra en el desarrollo de los primeros casos de estudio piloto, queda claro que inicialmente los alumnos no se muestran especialmente motivados por el uso de sistemas digitales que se alejan de las necesidades demandadas en las asignaturas más proyectuales. Es fácil determinar que los estudiantes consideran necesaria la tecnología tanto en cuanto en las principales materias esta es necesaria para desarrollar su trabajo. No obstante, el continuo avance de las TICs y los enfoques docentes, puede permitir utilizar sistemas como los juegos, la interacción o el render en tiempo real, en ámbitos como el diseño urbano o el diseño proyectual. La lógica reticencia inicial, queda superada cuando se pueden contemplar los resultados.

El artículo, constata esta evolución del estudiante, especialmente en cuanto a motivación. Mientras inicialmente la motivación se podía considerar media/baja, después de la realización del caso de estudio, se ha incrementado notablemente, aspecto que no solo refleja la utilidad del método, sino la potencialidad en la mejora académica y competencial del estudiante, la cual anteriormente ya ha quedado referenciada está ligada a la motivación del estudiante. El proyecto en sub-siguientes fases, mejorará la navegación e interacción del sistema para adaptarlo a las necesidades de cada zona urbana, todo ello introduciéndose cíclicamente en los contenidos de las materias para involucrar al alumno en proyectos reales.

\section{AgRADECIMIENTOS}

Esta investigación está financiada por el Programa Estatal de Investigación, Desarrollo e Innovación Orientada a los Retos de la Sociedad, en el marco del Plan Estatal de Investigación Científica y Técnica y de Innovación 20132016, bajo las referencias BIA2016-77464-C2-1-R (Gamificación para la enseñanza del diseño urbano y la integración en ella de la participación ciudadana, ArchGAME4CITY) y BIA2016-77464-C2-2-R (Diseño Gamificado de visualización 3D con sistemas de realidad virtual para el estudio de la mejora de competencias motivacionales, sociales y espaciales del usuario (EduGAME4CITY). (AEI/FEDER, UE).

\section{REFERENCIAS}

Bienkowski, M., Feng, M., \& Means, B. (2012). Enhancing teaching and learning through educational data mining and learning analytics: An issue brief. Washington, DC: SRI International, 1-57. https://doi.org/10.2991/icaiees13.2013.22

Boeykens, S., Santana Quintero, M., \& Neuckermans, H. (2008). Improving Architectural Design Analysis using 3D Modeling and Visualization techniques. In Digital Heritage: Proceedings of the 14th International Conference on Virtual Systems and Multimedia (pp. 6773). Retrieved from https://lirias.kuleuven.be/handle/123456789/202384

Fonseca, D., Martí, N., Redondo, E., Navarro, I., \& Sánchez, A. (2014). Relationship between student profile, tool use, participation, and academic performance with the use of Augmented Reality technology for visualized architecture models. Computers in Human Behavior. https://doi.org/10.1016/j.chb.2013.03.006

Fonseca, D., Redondo, E., \& Villagrasa, S. (2014). Mixedmethods research: a new approach to evaluating the motivation and satisfaction of university students using advanced visual technologies. Universal Access in the Information Society, 14(3), 311-332. https://doi.org/10.1007/s10209-014-0361-4

Fonseca, D., Valls, F., Redondo, E., \& Villagrasa, S. (2016). Informal interactions in 3D education: Citizenship participation and assessment of virtual urban proposals. Computers in Human Behavior. https://doi.org/10.1016/j.chb.2015.05.032

Hjorth, A., \& Wilensky, U. (2014). Redesigning Your City A Constructionist Environment for Urban Planning Education. Informatics in Education, 13(2), 197-208. https://doi.org/10.15388/infedu.2014.02

Intrinsic Motivation Inventory. (1994). Intrinsic Motivation Inventory (IMI). The Intrinsic Motivation Inventory, Scale Description, (Imi), 1-3. https://doi.org/www.selfdeterminationtheory.org

Kreijns, K., Van Acker, F., Vermeulen, M., \& Van Buuren, H. (2013). What stimulates teachers to integrate ICT in their pedagogical practices? the use of digital learning materials in education. Computers in Human Behavior, 29(1), 217-225. https://doi.org/10.1016/j.chb.2012.08.008

Oliver, R. (2002). The role of ICT in higher education for the 21st century: ICT as a change agent for education. In HE-21 Conference on the Role of ICT in Higher Education. https://doi.org/10.1080/09687760500376439

Peña, E., Fonseca, D., \& Martí, N. (2016). Relationship between learning indicators in the development and result of the building engineering degree final project. In ACM International Conference Proceeding Series (Vol. 02-04-Nove). https://doi.org/10.1145/3012430.3012537

Redondo, E., Valls, F., Fonseca, D., Navarro, I., Villagrasa, S., Olivares, A., \& Peredo, A. (2014). Educational qualitative assessment of augmented reality models and digital sketching applied to urban planning. In ACM International Conference Proceeding Series (Vol. Part F1092). https://doi.org/10.1145/2669711.2669938

Valls, F., Redondo, E., \& Fonseca, D. (2015). E-learning and serious games: New trends in architectural and urban design education. Lecture Notes in Computer Science (including subseries Lecture Notes in Artificial Intelligence and Lecture Notes in Bioinformatics). https://doi.org/10.1007/978-3-319-20609-7_59

Vicent, L., Villagrasa, S., Fonseca, D., \& Redondo, E. (2015). Virtual learning scenarios for qualitative assessment in higher education 3D arts. Journal of Universal Computer Science, 21(8), 1086-1105. 